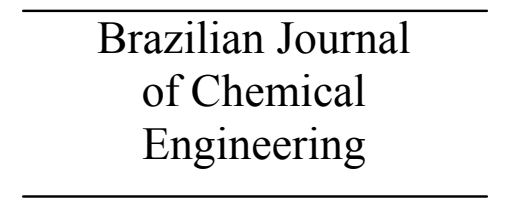

ISSN 0104-6632

Printed in Brazil

www.abeq.org.br/bjche

Vol. 28, No. 03, pp. 353 - 362, July - September, 2011

\title{
PREPARATION AND APPLICATION OF CHITOSAN NANOPARTICLES AND NANOFIBERS
}

\author{
Li-Ming Zhao ${ }^{1}$, Lu-E Shi ${ }^{2}$, Zhi-Liang Zhang ${ }^{2}$, Jian-Min Chen ${ }^{2}$, \\ Dong-Dong $\mathrm{Shi}^{2}$, Jie Yang ${ }^{2}$ and Zhen-Xing Tang ${ }^{1 *}$ \\ ${ }^{1}$ Department of Food Science and Technology, College of Biotechnology, Phone: +86-21-64250829, \\ East China University of Science and Technology, 200037, Shanghai, China. \\ E-mail: tangzhenxing@126.com \\ ${ }^{2}$ College of Life and Environmental Sciences, Hangzhou Normal University, 310036, \\ Hangzhou, Zhejiang, China.
}

(Submitted: February 23, 2011 ; Revised: March 25, 2011 ; Accepted: April 20, 2011)

\begin{abstract}
Encapsulation and immobilization technology is important for the food processing and bioengineering industries. Chitosan is a natural polysaccharide prepared by the $N$-deacetylation of chitin. It has been widely used in food and bioengineering industries, including the encapsulation of active food ingredients, in enzyme immobilization, and as a carrier for controlled drug delivery, due to its significant biological and chemical properties such as biodegradability, biocompatibility, bioactivity, and polycationicity. In this work, chitosan nanoparticles and nanofibers used to encapsulate bioactive substances and immobilize enzymes were reviewed. Preparation of chitosan nanoparticles and nanofibers, including the work achieved in our group on chitosan nanoparticles for enzyme immobilization, were also introduced. Some problems encountered with nano-structured chitosan carriers for bioactive substance encapsulation and enzyme immobilization were discussed, together with the future prospects of such systems.

Keywords: Chitosan; Encapsulation; Bioactive substances; Immobilization.
\end{abstract}

\section{INTRODUCTION}

Functional bioactive ingredients have received much attention in recent years from the scientific community, consumers and food manufacturers. Potential functional bioactive ingredients are vitamins, probiotics, bioactive peptides, antioxidants, etc. Scientific evidence to support the concept of health-promoting ingredients is growing steadily. Innovative functional foods that bring physiological benefits or reduce long-term risks of developing diseases are being developed by the scientific community (Elliott and Ong, 2002). However, due to the instability of functional ingredients under conditions encountered during processing (temperature, oxygen, light) or in the gut $(\mathrm{pH}$, enzymes, presence of other nutrients), it is difficult to retain the health benefits of functional ingredients (Bell, 2001). Therefore, it is necessary to protect these molecules from harsh conditions by encapsulation or immobilization.

Recently, various micro/nano carriers, particularly nanoparticles and nanofibers, have become available for enzyme immobilization (Jia et al., 2002; Kim and Grate, 2003; Lee et al., 2004; Sawicka et al., 2005; Wei et al., 2000). Typically, smaller particles provide a larger surface area for the attachment of enzymes (Jia et al., 2003) and a shorter diffusional path for the substrates. Thus, nano-structured carriers have been utilized as carriers for enzyme immobilization (Govardhan, 1999; Haring and Schreier, 1999; Xu et al., 1996; Kim et al., 2005).

*To whom correspondence should be addressed 
Chitosan is a partially deacetylated polymer of Nacetyl glucosamine that can be obtained through alkaline deacetylation of chitin. It consists of a $\beta$ $(1,4)$-linked-D-glucosamine residue with the amine groups randomly acetylated (Sevda and McClureb, 2004). The amine and $-\mathrm{OH}$ groups endow chitosan with many special properties, making it applicable in many areas and easily available for chemical reactions. Chitosan is safe, non-toxic and can interact with polyanions to form complexes and gels (Sunil et al., 2004; Se and Niranjan, 2005). In this work, the preparation of chitosan nanostructures and their application as bioactive ingredient encapsulators or immobilizers are reviewed.

\section{PREPARATION OF CHITOSAN NANOPARTICLES AND NANOFIBERS}

\section{Preparation of Chitosan Nanoparticles}

Nanoparticles are defined as particulate dispersions or solid particles with a size in the range of $1-1000 \mathrm{~nm}$. Methods, such as the emulsion method, ionic gelation method (Ohya et al., 1994; Yokohama et al., 1998; Kataoka et al., 2000), reverse micellar method, self-assembling method (Liu et al., 2005; Chen et al., 2003), etc., have been used to prepare chitosan nanoparticles. Here, the ionic gelation method and reverse micellar method are mainly considered.

\section{Ionic Gelation Method}

Chitosan nanoparticles can be prepared by the interaction of oppositely charged macromolecules. Tripolyphosphate (TPP) has often been used to prepare chitosan nanoparticles because TPP is nontoxic, multivalent and able to form gels through ionic interactions. The interaction can be controlled by the charge density of TPP and chitosan, which is dependent on the $\mathrm{pH}$ of the solution.

Nasti et al. (2009) studied the influence of a number of factors, such as $\mathrm{pH}$, concentration, ratios of components, and method of mixing, on the preparation of chitosan/TPP nanoparticles. Lin et al. (Lin et al., 2007) investigated the relationship between free amino groups on the surface and the characteristics of chitosan nanoparticles prepared by the ionic gelation method. These factors were unaffected by TPP concentration in these references.

A new and simple method to prepare magnetic $\mathrm{Fe}_{3} \mathrm{O}_{4}$-chitosan nanoparticles by cross-linking with TPP, precipitation with $\mathrm{NaOH}$ and oxidation with $\mathrm{O}_{2}$ in aqueous hydrochloric acid containing chitosan and $\mathrm{Fe}(\mathrm{OH})_{2}$ was developed by $\mathrm{Wu}$ et al. (Wu et al., 2009). Transmission electron microscopy (TEM) showed that the diameter of the magnetic $\mathrm{Fe}_{3} \mathrm{O}_{4}$ chitosan nanoparticles was $50-100 \mathrm{~nm}$. The capacity of lipase adsorption onto the nanoparticles could reach $129 \mathrm{mg} / \mathrm{g}$; and the maximal enzyme activity was $20.02 \mu \mathrm{mol} \mathrm{min}{ }^{-1} \mathrm{mg}^{-1}$ (protein) and activity retention was as high as $55.6 \%$ at $0.25 \mathrm{mg}$ protein $/ \mathrm{mg}$ nanoparticle concentration.

\section{Reverse Micellar Method}

Ultrafine polymeric nanoparticles with a narrow size range can be prepared with this method. The surfactant is dissolved in an organic solvent to prepare reverse micelles. An aqueous solution of chitosan is added with constant agitation to avoid any turbidity. The aqueous phase is regulated in such a way as to keep the entire mixture in an optically transparent microemulsion phase. More water should be added if nanoparticles of a larger size are to be prepared.

Brunel et al. (2008) used a reverse micellar method to prepare chitosan nanoparticles. The lower the molar mass of chitosan, the better the control over particle size and size distribution, probably as a result of either a reduction in the viscosity of the internal aqueous phase or an increase in the disentanglement of the polymer chains during the process.

Mansouri et al. (2010) prepared Bovine Serum Albumin (BSA)-loaded chitosan nanoparticles using the reverse micellar method. Particles were obtained in the size range of 143 to $428 \mathrm{~nm}$. The FTIR spectrum indicated that BSA was successfully encapsulated into the chitosan nanoparticles. The chitosan concentration and BSA loading played an important role in the release of BSA. Increasing the chitosan solution concentration decreased the release of BSA, both with a BSA loading of $10 \%$ and $20 \%$, while decreasing the BSA loading accelerated the release of BSA at either a chitosan concentration of $0.1 \%$ and $0.2 \%$.

Magnetic chitosan nanoparticles were prepared by a reversed phase suspension method using Span-80 as an emulsifier and glutaraldehyde as cross-linking reagent (Fang et al., 2009). Laccase was immobilized on the chitosan nanoparticles. The immobilized laccase exhibited an appreciable catalytic capability and had good storage stability and operation stability. The $\mathrm{K}_{\mathrm{m}}$ of immobilized and free laccase were 140.6 and $31.1 \mu \mathrm{M}$ in phosphate buffer $(0.1 \mathrm{M}, \mathrm{pH} 3.0)$, respectively. The immobilized laccase is a good candidate for the research and development of biosensors based on laccase catalysis. 


\section{Preparation of Chitosan Nanofibers}

Nanofibers are defined as solid particles with a dimension in the range of 1-1000 nm. Methods such as the sol-gel method, chemical vapor deposition method, electrospinning method, thermal oxidation method, etc., have been used to prepare nanofibers. However, the electrospinning method is mainly adopted to prepare nanofibers from nature resources. Therefore, electrospinning technology for chitosan nanofiber preparation is mainly discussed here.

Electrospinning technology for industrial use started in 1990. Since then, the study of electrospinning has shown that a large variety of polymers can be electrospun (Reneker and Chun, 1996). However, most processes to date focus on the electrospinning of synthetic polymers since the electrospinning of biopolymers is considerably more difficult due to the fact that biopolymers (polysaccharides and proteins) often have a distribution of molecular weights or have complex chemical structures. Viscosity is a key parameter in electrospinning because it is related to the extent that the polymer molecule chain is entangled within the solution (Ramakrishna et al., 2005). Electrospinning is also influenced by several other parameters, such as the operating voltage, the temperature, the pressure, and the flow rate. Of these parameters, the applied voltage is the most important one since it determines the degree of electrostatic interaction forces that induce the expulsion of a polymer jet. In some cases, an increase in fiber diameter can be observed with increased voltage (Jiang et al., 2007; Yao et al., 2007). Figure 1 illustrates the electrospinning setup.

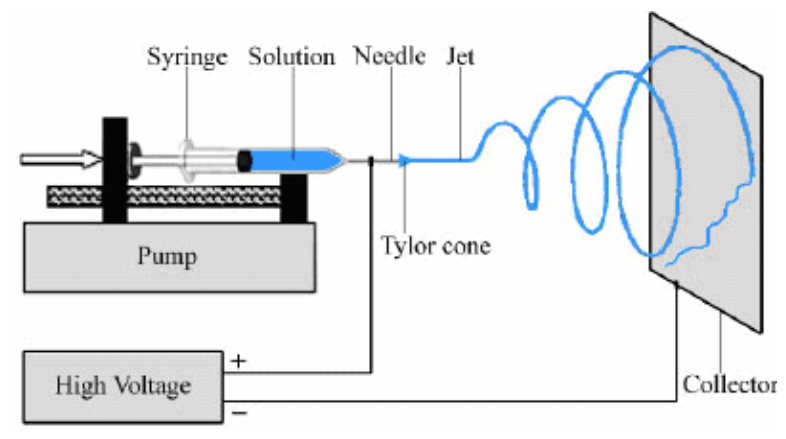

Figure 1: Electrospinning setup

An electrospun non-woven fabric of chitosan was successfully prepared by Ohkawa et al. (2004). Their study focused on the effects of the electrospinning solvent and the chitosan concentration on the morphology of the resulting non-woven fabrics. Among all solvents used, trifluoroacetic acid (TFA) was the most successful solvent system because the amino groups of chitosan form salts with TFA (Hasegawa et al., 1992), which can effectively destroy the rigid interactions between the chitosan molecules. As the chitosan concentration was increased, the morphology of the deposition on the collector changed from spherical beads to interconnected fibrous networks. Under optimized conditions, homogenous chitosan fibers with a mean diameter of $330 \mathrm{~nm}$ were obtained. Ohkawa et al. (2006) also optimized the viscosity of chitosan solutions (Baumgarten, 1971; Fridrikh et al., 2003) in order to decrease the average fiber diameter. It was determined that fiber diameter and polymer concentration have an inverse relationship.

Schiffman and Schauer (2007) reported that chitosans with a low (70 kDa), medium (190-310 kDa), and high (500 kDa) molecular weight, as well as a commercial chitosan (190-375 kDa) sample, were dispersed in $2.7 \%$ TFA. With TFA, all solutions formed uniform nanofibers with a diameter of $74 \pm$ $28 \mathrm{~nm}, 77 \pm 29 \mathrm{~nm}, 108 \pm 42 \mathrm{~nm}$, and $58 \pm 20 \mathrm{~nm}$ for low, medium, and high molecular weight, and commercial grade chitosan, respectively. The higher molecular weight chitosan produced larger fibers. Subsequent crosslinking of chitosan nanofibers with glutaraldehyde led to a substantial increase in the fiber size for all chitosans, e.g., the mean diameter of fibers for low, medium, and high molecular weight, and commercial chitosan increased to $387 \pm 183 \mathrm{~nm}$, $172 \pm 75 \mathrm{~nm}, 137 \pm 59 \mathrm{~nm}$, and $261 \pm 160 \mathrm{~nm}$, respectively. This illustrated how the processing parameters may be adjusted to find the most suitable working conditions to produce nanofibers efficiently and effectively.

Torres-Giner et al. (2008a) developed electrospun chitosan nanofibers using TFA and dichloromethane as solvent. In addition, Torres-Giner et al. (2008b) also developed porous electrospun chitosan nanofibers using pure trichloromethane as solvent. Geng et al. (2005) reported the manufacture of chitosan nanofibers in concentrated aqueous acetic acid solutions of varying acetic acid concentration. Chitosan of low (30 kDa), medium (106 kDa), and high molecular weight $(398 \mathrm{kDa})$ formed beads and thin fibers $(40 \mathrm{~nm})$ at an acetic acid concentration of $30 \%$. Of all the polymers and polymer concentrations tested, only $106 \mathrm{kDa}$ chitosan produced bead-free fibers with an average diameter of $130 \mathrm{~nm}$ from 7\% solutions. The low molecular weight chitosan solutions of 9.5-10\% yielded large beads and very fragile fibers, while the high molecular weight chitosan solutions of $2.5-3 \%$ produced very fine and fragile fibers with rough surfaces and an average diameter of $60 \mathrm{~nm}$. This 
highlights the need to balance the molecular weight and the polymer concentration requirements such that sufficient polymer chain entanglement occurs while maintaining a low enough viscosity to allow the processing of the solution. The solvent type plays a crucial role in modulating both the entanglement and the solution viscosity.

Chitosan nanofibers were also produced by the electrospinning of chitin solutions using 1,1,1,3,3,3hexafluoro-2-propanol (HFIP) as the spinning solvent (Min et al., 2004). Fiber diameters ranged between 50 and $460 \mathrm{~nm}$, but most fibers were smaller than $200 \mathrm{~nm}$. Subsequent deacetylation in sodium hydroxide yielded chitosan fibers with a degree of deacetylation of approximately $85 \%$. The deacetylation apparently had no significant influence on the morphology and the fiber diameter.

\section{ENCAPSULATION OF BIOACTIVE INGREDIENTS}

Chitosan-based systems have wide and rapidly increasing applications in the food and biochemical industries. Virtually any ingredients can be encapsulated, whether hydrophobic, hydrophilic, or even bacterial. The following section discusses some chitosan nanosystems for bioactive ingredient encapsulation and immobilization. Methods used to prepare chitosan microparticles can also be used for chitosan nanoparticle preparation. Thus, some chitosan microsystems for bioactive ingredient encapsulation and immobilization are also discussed.

\section{Vitamins}

Chen and Subirade (2005) prepared core-shell nanoparticles $\quad(100-200 \quad \mathrm{~nm}$ hydrodynamic diameters) for delivery of riboflavin by crosslinking chitosan with sodium tripolyphosphate (TPP), followed by coating the nano-gel-particles with either native or denatured 3-lactoglobulin.

Sustained release carriers of vitamin $\mathrm{C}$ were prepared with cross-linked chitosan as a wall material by a spray-drying technique (Desai and Park, 2005; Desai and Park, 2006; Desai et al., 2006). Vitamin C-encapsulated chitosan microspheres of different size, surface morphology, loading efficiency, and zeta potential with controlled-release properties could be obtained by varying the manufacturing parameters (inlet temperature, flow rate) and using different molecular weights and concentrations of chitosan. Vitamin C-encapsulated chitosan microcapsules were spherical in shape with a smooth surface, as observed by scanning electron microscopy.

Jiang and Lee (2008) investigated the stability and characteristics of vitamin C-loaded chitosan nanoparticles during heat processing in aqueous solutions. Vitamin C-loaded chitosan nanoparticles were prepared by ionic gelation of chitosan with TPP anions. The smallest chitosan nanoparticles $(170 \mathrm{~nm})$ were obtained with a chitosan concentration of $1.5 \mathrm{mg} / \mathrm{mL}$ and a TPP concentration of $0.6 \mathrm{mg} / \mathrm{mL}$. At the beginning of the release profiles, the burst release-related stability of the surface increased with temperature. Then, the release of the internal vitamin $\mathrm{C}$ was constantly higher with a longer release time. Consequently, it was confirmed that the stability of vitamin C-loaded chitosan nanoparticles was affected by temperature, but that the internal stability was greater than the surface stability. These results demonstrated the stability of chitosan nanoparticles during heat processing and suggested the possible use of vitamin C-loaded chitosan nanoparticles to enhance antioxidant effects because of the continuous release of vitamin $\mathrm{C}$ from chitosan nanoparticles in food processing.

\section{Probiotics}

Probiotics can be defined as living microbial supplements that can improve the balance of intestinal microorganisms (Fuller, 1992). Good probiotic viability and activity are considered to be essential for optimal functionality (Mattila-Sandholm et al., 2002; Champagne and Gardner, 2005). Coating alginate beads with chitosan has been studied extensively for encapsulating probiotics (Cui et al., 2000; Canh et al., 2004; Smidsrod and SkjakBraek, 1990). Sprayed particles coated with chitosan are also recommended as impressively effective vehicles for delivering viable bacterial cells to the colon and are stable shells during refrigerated storage. Krasaekoopt et al. (2004) compared the survival of microencapsulated probiotics using different coating materials and found that chitosancoated alginate beads provided better protection for Lactobacillus acidophilus and Lactobacillus casei than did poly-L-lysine-coated alginate beads in $0.6 \%$ bile salts. Moreover, chitosan-coated alginate beads provided the best protection for the strains $L$. acidophilus 547 and L. casei 01 . Krasaekoopt et al. (Krasaekoopt et al., 2006) also studied the survival of probiotics encapsulated in chitosan-coated alginate beads in yogurt and found that the survival of the encapsulated probiotic bacteria was higher than for free cells by approximately a factor of ten. 
Zhou et al. (1998) reported that suspending alginate capsules in a low molecular weight chitosan solution reduced cell release by $40 \%$. Low molecular weight chitosan diffused more readily into the calcium alginate gel matrix, resulting in a denser membrane than with high molecular weight chitosan (McKnight et al., 1988).

Bifidobacterium animalis subsp. lactis was entrapped in alginate (as control), alginate-chitosan, alginate-chitosan-Sureteric and alginate-chitosanAcryl-Eze (Liserre et al., 2007). Survival and in vitro release of bifidobacteria from the microparticles were investigated under conditions simulating gastrointestinal fluids covering the $\mathrm{pH}$ range from 1.5 to 7.5 , with and without pepsin, pancreatin, and bile. All types of microcapsules protected $B$. animalis, but the use of chitosan and enteric polymers in the formulation of the beads, especially Acryl-Eze, enhanced the beneficial effects of the microencapsulation technique. Besides promoting the controlled release of bifidobacteria in simulated gastrointestinal juices, the microencapsulation with enteric polymers improved the survival rate of these microorganisms.

\section{Unsaturated Fatty Acids}

Unsaturated fatty acids are important nutrients involved in many body functions, including neuro-protective, antioxidant, and anti-inflammatory effects and cardiovascular health. However, their development as nutritional supplements is limited by their high susceptibility to oxidative rancidity. Chitosan is very effective at stabilizing oil-in-water emulsions by adsorbing to the oil droplet surface, lowering interfacial tension and forming protective membranes around the lipid.

Klinkesorn et al. (2005) investigated the physicochemical and oxidative stability of spraydried tuna oil-in-water emulsion droplets with a coating of chitosan and lecithin multilayer systems. Their results showed that spray-dried tuna oil-inwater emulsions stabilized by chitosan-lecithin membranes were more oxidatively stable than buck oils and thus have excellent potential as $\omega-3$ fatty acid ingredients for functional foods. Shaw et al. (2007) also investigated the stability of spray-dried multilayer emulsion. Lecithin-chitosan multilayered emulsions were spray-dried with corn syrup solids. These studies suggested that a microencapsulated multilayered emulsion system could be used as a delivery system for improved oxidative stability.

Park et al. (2007) investigated the influence of interfacial composition on the in vivo digestibility of lipids coated with chitosan. Their results suggested that encapsulation of lipids by chitosan did not inhibit their in vivo digestibility. Consequently, it should be possible to use chitosan to microencapsulate lipids and lipid-soluble components without compromising their bioavailability, although further human studies are needed to confirm this.

Bustos et al. (2003) demonstrated the feasibility of using an emulsion phase separation technique involving an ionotropic gelation method to prepare chitosan microcapsules stabilizing fish oil such as krill oil, which is rich in both eicosapentaenoic acid (EPA) and docosahexaenoic acid (DHA). Emulsions were prepared from a mixture of chitosan, Tween 20 as an emulsifier and krill oil and then slowly added into a stirred solution of sodium hydroxide in ethanol for hardening of the wall matrix of the dispersed emulsion particles. Chitosan microcapsules thus prepared were reported not to be significantly effective in stabilizing the encapsulated krill oil against oxidation compared to the corresponding emulsion form, suggesting the need for some modifications of the properties of the wall materials to improve oxidative stability.

\section{Phytochemicals}

Phytochemicals have received much attention in recent years due to their potential for lowering blood pressure, reducing cancer risk factors, regulating digestive tract activity, strengthening immune systems, regulating growth, controlling blood sugar concentration, lowering cholesterol levels and serving as antioxidants (Wildman and Kelly, 2001).

$\mathrm{Hu}$ et al. (2008) investigated the process of fabricating chitosan-TPP nanoparticles intended to be used as carriers for delivering tea catechins. The effects of modulating conditions, including contact time between chitosan and tea catechins, molecular mass, chitosan concentration, chitosan-TPP mass ratio, initial $\mathrm{pH}$ value of the chitosan solution, and concentration of tea catechins, on the encapsulation efficiency and the release profile of tea catechins in vitro were examined systematically. The study found that the encapsulation efficiency of tea catechins in chitosan-TPP nanoparticles ranged from 24 to $53 \%$. In addition, FT-IR analysis showed that covalent bonding and hydrogen bonding between tea catechins and chitosan occurred during the formation of chitosan-TPP nanoparticles loaded with tea catechins. Furthermore, studies of the release profile of tea catechins in vitro demonstrated that the controlled release of tea catechins was achievable using chitosan-TPP nanoparticles. 
Kittikaiwan et al. (2007) developed a novel encapsulation method using chitosan (with 80\% deacethylation) to encapsulate Haematococcus pluvialis, a rich source of astaxanthin. $H$. pluvialis beads were coated five times with chitosan solution, forming multilayer films of chitosan. Chitosan capsules showed uniform shape with diameters from 0.35 to $0.5 \mathrm{~cm}$ and $100 \mathrm{~mm}$ film thickness. Storage studies of chemical stability proved that chitosan as a wall material can prevent degradation of astaxanthin. Higuera-Ciapara et al. (2004) used a solvent evaporation method to encapsulate astaxathin in a chitosan matrix cross-linked with glutaraldehyde. Microcapsules were chemically stable over a storage time of eight weeks.

\section{Flavors}

Hsieh et al. (2006) emulsified citronella oil in the presence of $0.2 \%$ chitosan and increased the $\mathrm{pH}$ with sodium hydroxide to form a shell of chitosan around the emulsion droplets. After washing twice with distilled water, the dispersion of the encapsulates improved the aroma released out of dried microcapsules within a few hours (depending on the temperature).

Djordjevic et al. (2007) examined whether citral and limonene were more stable in emulsions stabilized with a sodium dodecyl sulfate (SDS)chitosan complex than with gum arabic (GAR). Citral degraded less in GAR-stabilized emulsions than in SDS-chitosan-stabilized emulsions at pH 3.0. However, SDS-chitosan-stabilized emulsions were more effective at retarding the formation of the citral oxidation product than GAR stabilized emulsions. Limonene degradation and formation of the limonene oxidation products, limonene oxide and carvone, were lower in the SDS-chitosan- than in the GAR-stabilized emulsions at pH 3.0. The ability of an SDS-chitosan multilayer emulsifier system to inhibit the oxidative deterioration of citral and limonene could be due to the formation of a thick cationic emulsion droplet interface that can repel prooxidative metals, thus decreasing prooxidant-lipid interactions.

\section{Enzymes}

The immobilization of enzymes has proven to be particularly valuable, because it allows enzymes to be easily reused multiple times for the same reaction with longer half-lives and less degradation. It has also helped to prevent the contamination of the substrate with enzyme/protein or other compounds, which decreases purification costs. These benefits of immobilized enzymes have made them highly applicable in a range of evolving biotechnologies (Spahn and Minteer, 2008).

Biro et al. (2008) prepared macro-, micro- and nanosized chitosan particles suitable as immobilization carriers by precipitation, emulsion cross-linking and ionic gelation methods, respectively. Activities of $\beta$-galactosidase covalently attached to differently sized particles were evaluated and compared. The highest activity was shown by the biocatalyst immobilized on nanoparticles obtained by means of the ionotropic gelation method with sodium sulfate as a gelation agent. $\beta$-Galactosidase fixed on macro- and microspheres exhibited excellent storage stability in aqueous solution, with no more than 5\% loss of activity after three weeks storage at $4^{\circ} \mathrm{C}$ and $\mathrm{pH}$ 7.0.

Liu et al. (2005) studied trypsin immobilized on linolenic acid-modified chitosan nanoparticles using glutaraldehyde (GA) as cross-linker. Their results indicated that the activity of trypsin immobilized on linolenic acid modified chitosan nanoparticles increased with increasing concentration of GA up to $0.07 \%(\mathrm{v} / \mathrm{v})$ and then decreased with increasing amounts of GA. On the other hand, particle size increased (from 523 to $1372 \mathrm{~nm}$ ) with increasing concentration of GA (from 0.03 to $0.1 \% \mathrm{v} / \mathrm{v}$ ). The kinetic constant value $\left(\mathrm{K}_{\mathrm{m}}\right)$ of trypsin immobilized on nanoparticles $(71.9 \mathrm{mg} / \mathrm{mL})$ was higher than that of pure trypsin $(50.2 \mathrm{mg} / \mathrm{mL})$. However, the thermal stability and optimum temperature of trypsin immobilized on nanoparticles improved, which made it more attractive for applications.

Sjoholm et al. (2009) evaluated the effect that the degree of deacetylation has on the hydrophobic modification of medium molecular weight chitosan via reductive amination with long chain aldehydes and the resulting changes in enzyme activity after the immobilization of glucose oxidase in the polymeric micellar structure. Results suggested that a high degree of deacetylation provided optimal enzyme immobilization properties (i.e., high activity).

\section{OUR WORK ON CHITOSAN NANOPARTICLES}

Reverse micellar and ionic gelatation methods were used to prepare chitosan nanoparticles in our group. Figure 2 presents scanning electron micrographs of nanoparticles prepared by different methods. The nanoparticles were about $50-80 \mathrm{~nm}$ in size. However, in Figure 1B some oil phase is seen, which indicated that the product was washed insufficiently. 
Based on our results, solution $\mathrm{pH}$, TPP concentration, and chitosan concentration significantly affected the size of the chitosan nanoparticles. Response surface methodology was employed to optimize the size of chitosan nanoparticles. The adequacy of the model equation for predicting the order of magnitude of the size of the chitosan nanoparticles was verified using validation data. The minimum particle size was about $42 \pm 5 \mathrm{~nm}$ under the optimized conditions (Tang et al., 2007).

Neutral proteinase and neclease P1 were immobilized on chitosan nanoparticles, respectively (Tang et al., 2006; Shi and Tang, 2009). The optimal conditions for immobilization were as follows: One milligram of neutral proteinase was immobilized on chitosan nanoparticles for about $15 \mathrm{~min}$ at $40^{\circ} \mathrm{C}$.
Under the optimized conditions, the enzyme activity yield was $84.3 \%$. The enzyme exhibited remarkably improved properties, such as thermal stability, reuse, and storage stability after it had been immobilized on chitosan nanoparticles.

Sorption kinetics and the sorption mechanism for neutral lipase were studied (Tang et al., 2007). Adsorption of neutral lipase on chitosan nanoparticles fit the Lagergren first-order equation at an initial neutral lipase concentration of $3.0 \mathrm{mg} / \mathrm{mL}(\mathrm{pH} \mathrm{7.0)}$ ) The firstorder constant for neutral lipase was $23.34 \mathrm{~h}^{-1}$. When the neutral lipase concentration was controlled in a certain region, it fit the Freundlich isothermal linear equation. According to IR spectral analysis, hydrogen bonding might be the main force between the hydroxyl group, $\mathrm{NH}_{2}$ group and neutral lipase (Figure 3).
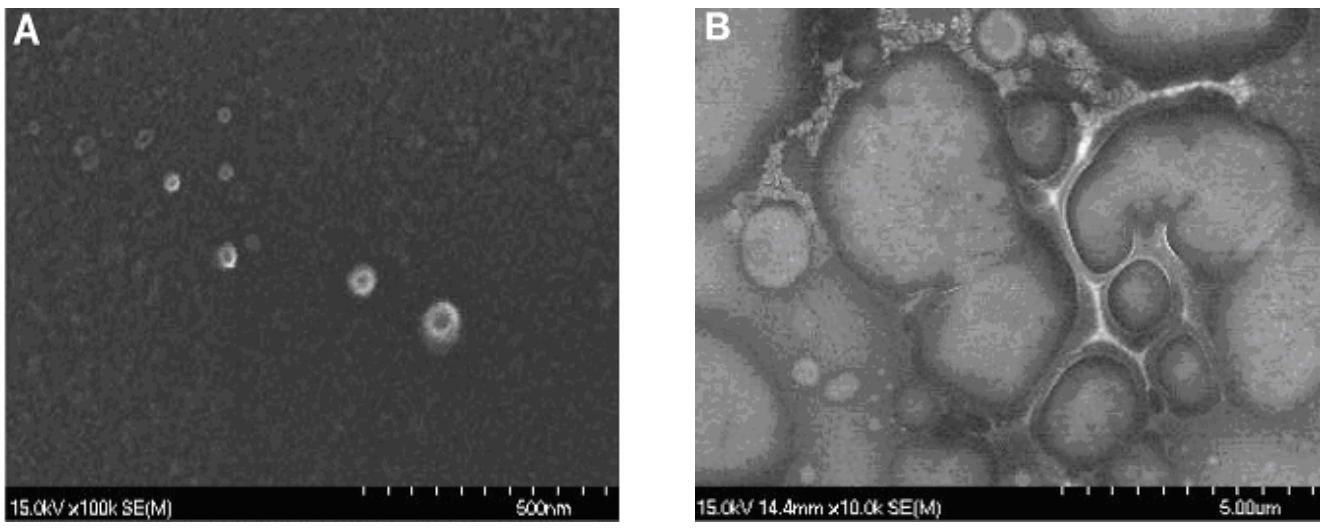

Figure 2: Scanning electron micrographs of (A) nanoparticles made by the ionic gelatation method and (B) nanoparticles made by the reverse micelle method.
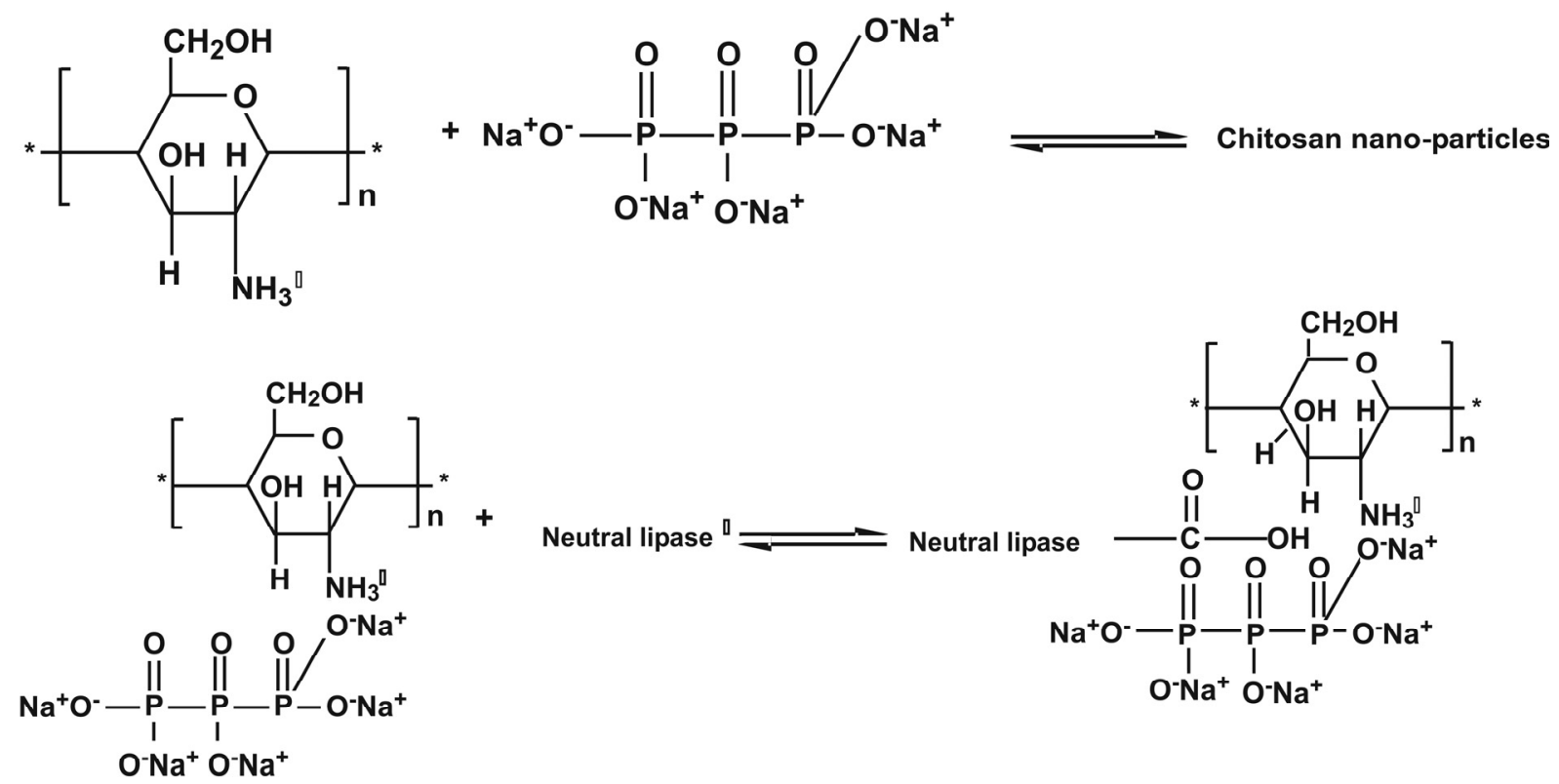

Figure 3: Hypothesis for the adsorption mechanism. 


\section{CONCLUSIONS}

This review summarized the preparation of chitosan nanostructures and their application for encapsulating and immobilizing bioactive ingredients. From this review, it is concluded that micro/nanostructured chitosans can be used as bioactive ingredients carriers. They have the potential for the development of novel encapsulation or immobilization carriers. Due to their favorable biological properties such as non-toxicity, biocompatibility, biodegradability and antibacterial ability, they are also promising candidates for drug delivery carriers and cell proliferation enhancers. However, most of these studies are still at the laboratory level. Additional studies are necessary before their industrial application. We hope that more chitosan-based nanocarriers can be developed and applied in the biochemical and food engineering fields in the near future.

\section{REFERENCES}

Baumgarten P. K., Electrostatic spinning of acrylic microfibers. J. Colloid Interface Sci., 36, 71-79 (1971).

Bell, L. N., Handbook of Nutraceuticals and Functional Foods. New York, USA (2001).

Biró, E., Németh, Á. S., Sisak, C., Feczkó, T. and Gyenis, J., Preparation of chitosan particles suitable for enzyme immobilization. J. Biochem. Biophys. Methods, 70, 1240-1246 (2008).

Brunel, F., Veron, L., David, L., Domard, A. and Delair, T., A Novel synthesis of chitosan nanoparticles in reverse emulsion. Langmuir, 24, 11370-11377 (2008).

Bustos, R., Romo, L., Yáñez, K., Díaz, G. and Romo, C., Oxidative stability of carotenoid pigments and polyunsaturated fatty acids in microparticulate diets containing krill oil for nutrition of marine fish larvae. J. Food Eng., 56, 289-293 (2003).

Canh, L., Millette, M., Mateescu, M. and Lacroix, M., Modified alginate and chitosan for lactic acid bacteria immobilization. Biotechnol. Appl. Biochem., 39, 347-354 (2004).

Champagne, C. P. and Gardner, N. J., Challenges in the addition of probiotic cultures to foods. Crit. Rev. Food Sci. Nutr., 45, 61-84 (2005).

Chen, L. Y. and Subirade, M., Chitosan/ß-lactoglobulin core-shell nanoparticles as nutraceutical carriers. Biomaterials, 26, 6041-6053 (2005).

Chen, X. G., Lee, C. M. and Park, H. J., O/W emulsification for the self-aggregation and nanoparticle formation of linoleic acids modified chitosan in the aqueous system. J. Agric. Food Chem., 51, 3135-3139 (2003).

Cui, J., Goh, J., Kim, P., Choi, S. and Lee, B., Survival and stability of Bifidobacteria loaded in alginate poly-Llysine microparticles. Int. J. Pharm., 210, 51-59 (2000).

Desai, K. G. H., Liu, C. and Park, H. J., Characteristics of vitamin $\mathrm{C}$ encapsulated tripolyphosphate-chitosan microspheres as affected by chitosan molecular weight. J. Microencapsulation, 23, 79-90 (2006).

Desai, K. G. H. and Park, H. J., Effect of manufacturing parameters on the characteristics of vitamin $\mathrm{C}$ encapsulated tripolyphosphatechitosan microspheres prepared by spray drying. J. Microencapsulation, 23, 91-103 (2006).

Desai, K. G. H. and Park, H. J., Encapsulation of vitamin $\mathrm{C}$ in tripolyphosphate crosslinked chitosan microspheres by spray drying. J. Microencapsulation, 22, 179-192 (2005).

Djordjevic, D., Cercaci, L., Alamed, J., McClements, D. J. and Derker, E. A., Chemical and physical stability of citral and limonene in sodium dodecyl sulphate-chitosan and gum Arabic-stabilized oilin-water emulsions. J. Agric. Food Chem., 55, 3585-3591 (2007).

Elliott, R. and Ong, T. J., Science, medicine, and the future of nutritional genomics. Br. Med. J., 324, 1438-1442 (2002).

Fang, H., Huang, J., Ding, L. Y., Li, M. T. and Chen, Z., Preparation of magnetic chitosan nanoparticles and immobilization of laccase. J. Wuhan Univ. Technol., 24, 42-47 (2009).

Fridrikh, S. V., Yu, J. H., Brenner, M. P. and Rutledge, G. C., Controlling the fiber diameter during electrospinning. Phys. Rev. Lett., 90, 144502 (2003).

Fuller, R., Probiotics: The Scientific Basis. London, UK (1992).

Geng, X. Y., Kwon, O. H. and Jiang, J. H., Electrospinning of chitosan dissolved in concentrated acetic acid solution. Biomaterials, 26, 5427-5432 (2005).

Govardhan, C. P., Crosslinking of enzymes for improved stability and performance. Curr. Opin. Biotechnol., 10, 331-335 (1999).

Haring, D. and Schreier, P., Cross-linked enzyme crystals. Curr. Opin. Chem. Biol., 3, 35-38 (1999).

Hasegawa, M., Isogai, A., Onabe, F., Usuda, M. and Atalla, R.H., Characterization of cellulosechitosan blend films. J. Appl. Polym. Sci., 45, 1873-1879 (1992).

Higuera-Ciapara, I., Felix-Valenzuela, L., Goycoolea, F. M. and Argüelles-Monal, W., Microencapsulation 
of astaxanthin in a chitosan matrix. Carbohydr. Polym., 56, 41-45 (2004).

Hsieh, W. C., Chang, C. P. and Gao, Y. L., Controlled release properties of chitosan encapsulated volatile citronella oil microcapsules by thermal treatments. Colloids Surf. B., 53, 209214 (2006).

Hu, B., Pan, C., Sun, Y., Hou, Z., Ye, H., Hu, B. and Zeng, X. X., Optimization of fabrication parameters to produce chitosan-tripolyphosphate nanoparticles for delivery of tea catechins. J. Agric. Food Chem., 56, 7451-7458 (2008).

Jang, K. I. and Lee, H. G., Stability of Chitosan Nanoparticles for 1-Ascorbic Acid during Heat Treatment in Aqueous Solution. J. Agric. Food Chem., 56, 1936-1941 (2008).

Jia, H. F., Zhu, G. Y., Vugrinovich, B., Kataphinan, W., Reneker, D. H. and Wang, P., Enzymecarrying polymeric nanofibers prepared via electrospinning for use as unique biocatalysts. Biotechnol. Prog., 18, 1027-1032 (2002).

Jia, H., Zhu, G. and Wang, P., Catalytic behaviors of enzymes attached to nanoparticles; the effect of particle mobility. Biotechnol. Bioeng., 84, 406-414 (2003).

Jiang, H. L., Zhao, P. C. and Zhu, K. J., Fabrication and characterization of zein-based nanofibrous scaffolds by an electrospinning method. Macromol. Biosci., 7, 517-525 (2007).

Kataoka, K., Matsumoto, T., Tokohama, M., Okano, T. and Sakurai, Y., Doxorubicin-loaded poly(ethylene glycol)-poly(b-benzyl-L-aspartate) copolymer micelles: their pharmaceutical characteristics and biological significance. J. Controlled Release, 64, 143-153 (2000).

Kim, B. C., Nair, S., Kim, J., Kwak, J. H., Grate, J. W., Kim, S. H. and Gu, M. B., Preparation of biocatalytic nanofibers with high activity and stability via enzyme aggregate coating on polymer nanofibers. Nanotechnolgy, 16, S382S388 (2005).

Kim, J. and Grate, J. W., Single-enzyme nanoparticles armoured by a nanometer-scale organic/inorganic network. Nano Lett., 3, 1219-1222 (2003).

Kittikaiwan, P., Powthongsook, S., Pavasant, P. and Shotipruk, A., Encapsulation of Haematococcus pluvialis using chitosan for astaxanthin stability enhancement. Carbohydr. Polym., 70, 378-385 (2007).

Klinkerson, U., Sophanodora, P., Chinachoti, P., McClements, D. J. and Decker, E. A., Stability of spray-dried tuna oil emulsions encapsulated with two-layered interfacial membranes. J. Agric. Food Chem., 53, 8365-8371 (2005).
Krasaekoopt, W., Bhandari, B. and Deeth, H. C., Survival of probiotics encapsulated in chitosancoated alginate beads in yoghurt from UHT- and conventionally treated milk during storage. LWTFood Sci. Technol., 39, 177-183 (2006).

Krasaekoopt, W., Bhandari, B. and Deeth, H., The influence of coating materials on some properties of alginate beads and survivability of microencapsulated probiotic bacteria. Int. Dairy J., 14, 737-743 (2004).

Lee, J., Han, S. and Hyeon, T., Synthesis of new nanoporous carbon materials using nanostructured silica materials as templates. J. Mater. Sci., 14, 478-486 (2004).

Lin, A. H., Liu, Y. M. and Ping, Q. N., Free amino groups on the surface of chitosan nanoparticles and its characteristics. Yao Xue Xue Bao., 42, 323-328 (2007).

Liserre, A. M., Re, M. I., Franco, B. D. G. M., Microencapsulation of bifidobacterium animalis subsp. Lactis in modified alginate-chitosan beads and evaluation of survival in simulated gastrointestinal conditions. Food Biotechnol., 21, $1-16$ (2007).

Liu, C. G., Chen, X. G. and Park, H. J., Selfassembled nanoparticles based on linoleic-acid modified chitosan: stability and adsorption of trypsin. Carbohydr. Polym., 62, 293-298 (2005).

Liu, C. G., Desai, K. G. H., Chen, X. G. and Park, H. J., Preparation and characterization of nanoparticles containing trypsin based on hydrophobically modified chitosan. J. Agric. Food Chem., 53, 17281733 (2005).

Mansouri, M., Khorram, M., Samimi, A. and Osfouri, S., Preparation of bovine serum albumin loaded chitosan nanoparticles using reverse micelle method. http://www.chisa.cz/2010/admin/contrib_get_abst ract.asp?id_02 $=942$.

Mattila-Sandholm, T., Myllärinen, P., Crittenden, R., Mogensen, G., Fonden, R., and Saarela, M., Technological challenges for future probiotic foods. Int. Dairy J., 12, 173-182 (2002).

McKnight, C. A., Ku, A. and Goosen, M. F., Synthesis of chitosan-alginate microencapsule membrane. J. Bioact. Compat. Polym., 3, 334-354 (1988).

Min, B. M., Lee, S. W., Lim, J. N., You, Y., Lee, T. S. and Kang, P. H., Chitin and chitosan nanofibers: electrospinning of chitin and deacetylation of chitin nanofibers. Polymer., 45, 7137-7142 (2004).

Nasti, A., Zaki, N. M., Leonardis, P. D., Ungphaiboon, S., Sansongsak, P., Rimoli, M. G. and Tirelli, N., Chitosan/TPP and chtiosan/TPP-hyaluronic acid nanoparticles: systematic optimization of the 
preparative process and preliminary biological evaluation. Pharm. Res., 26, 1918-1930 (2009).

Ohkawa, K., Cha, D. I., Kim, H., Nishida, A. and Yamamoto, H., Electrospinning of chitosan. Macromol. Rapid Commun., 25, 1600-1605 (2004).

Ohkawa, K., Minato, K. I., Kumagai, G., Hayashi, S. and Yamamoto, H., Chitosan nanofiber. Biomacromolecules, 7, 3291-3294 (2006).

Ohya, Y., Shiratani, M., Kobayashi, H. and Ouchi, T., Release behavior of 5-fluorouracil from chitosan-gel nanospheres immobilizing 5fluorouracil coated with polysaccharides and their cell specific cytotoxicity. Pure Appl. Chem., 31, 629-642 (1994).

Park, G. Y., Mun, S., Park, Y., Rhee, S., Decker, E. A., Weiss, J., McClements, D. J. and Park, Y., Influence of encapsulation of emulsified lipids with chitosan on their in vivo digestibility. Food Chem., 104, 761-767 (2007).

Ramakrishna, S., Fujihara, K., Teo, W. E., Lim, T. C. and Ma, Z. H., An Introduction to Electrospinning and Nanofibers. Singapore (2005).

Reneker, D. H. and Chun, I., Nanometre diameter fibres of polymer, produced by electrospinning. Nanotechnology., 7, 216-223 (1996).

Sawicka, K., Gouma, P. and Simon, S., Electrospun biocomposite nanofibers for urea biosensing. Sens. Actuators, B., 108, 585-588 (2005).

Schiffman, J. D. and Schauer, C. L., One-step electrospinning of crosslinked chitosan fibers. Biomacromolecules., 8, 2665-2667 (2007).

Sevda, S. and McClureb, S. J., Potential applications of chitosan in veterinary medicine. Adv. Drug Delivery Rev., 56, 1467-1480 (2004).

Se, K. K. and Niranjan, R., Enzymatic production and biological activities of chitosan oligosaccharides (COS): a review. Carbohydr. Polym., 62, 357-368 (2005).

Shaw, L. A., McClements, D. J. and Decker, E. A., Spray-dried multilayered emulsions as a delivery method for $\omega-3$ fatty acids into food systems. J. Agric. Food Chem., 55, 3112-3119 (2007).

Shi, L. E. and Tang, Z. X., Adsorption of nuclease p1 on chitosan nano-particles. Braz. J. Chem. Eng., 26, 435-443 (2009).

Sjoholm, K. H., Cooney, M. and Minteer, S. D., Effects of degree of deacetylation on enzyme in hydrophobically modified chitosan. Carbohydr. Polym., 77, 420-424 (2009).

Smidsrod, O. and Skjak-Braek, G., Alginate as immobilization matrix for cells. Trends Biotechnol., 8, 71-78 (1990).

Spahn, C. and Minteer, S. D., Enzyme immobilization in biotechnology. Recent Pat. Eng., 2, 195-200 (2008).
Sunil, A. A., Nadagouda, N. M. and Tejraj, M., Recent advances on chitosan-based micro- and nanoparticles in drug delivery. J. Controlled Release, 100, 5-28 (2004).

Tang, Z. X., Qian, J. Q. and Shi, L. E., Characterizations of immobilized neutral proteinase on chitosan nano-particles. Process Biochem., 41, 1193-1197 (2006).

Tang, Z. X., Shi, L. E. and Qian, J. Q., Neutral lipase from aqueous solutions on chitosan nanoparticles. Biochem. Eng. J., 34, 217-223 (2007).

Tang, Z. X., Qian, J. Q. and Shi, L. E., Preparation of chitosan nanoparticles as carrier for immobilized enzyme. Appl. Biochem. Biotechnol., 136, 77-96 (2007).

Torres-Giner, S., Gimenez, E. and Lagaron, J. M., Characterization of the morphology and thermal properties of Zein Prolamine nanostructures obtained by electrospinning. Food Hydrocolloids., 22, 601-14 (2008a).

Torres-Giner, S., Ocio, M. J. and Lagaron, J. M., Development of active antimicrobial fiber based chitosan polysaccharide nanostructures by electrospinning. Eng. Life Sci., 8, 303-314 (2008b).

Wei, Y., Xu, J., Feng, Q., Dong, H. and Lin, M., Encapsulation of enzymes in mesoporous host materials via the nonsurfactant-templated sol-gel process. Mater. Lett., 44, 6-11 (2000).

Wildman, R. E. C. and Kelly, M., Handbook of Nutraceuticals and Functional Foods. New York, USA (2001).

Wu, Y., Wang, Y. J., Luo, G. S. and Dai, Y. Y., In situ preparation of magnetic chitosan nanoparticles for lipase immobilization by cross-linking and oxidation in aqueous solution. Bioresour. Technol., 100, 3459-3464 (2009).

$\mathrm{Xu}, \mathrm{K}$. and Klibanov, A. M., pH control of the catalytic activity of crosslinked enzyme crystals in organic solvents. J. Am. Chem. Soc., 118, 9815-9819 (1996).

Yao, C., Li, X. S. and Song, T. Y., Electrospinning and crossfinking of Zein nanofiber mats. J. Appl. Polym. Sci., 103, 380-385 (2007).

Yokohama, M., Fukushima, S., Uehara, R., Okamoto, K. and Kataoka, K., Characterization of physical entrapment and chemical conjugation of adriamycin in polymeric micelles and their design for in vivo delivery to a solid tumor. J. Controlled Release, 50, 79-92 (1998).

Zhou, Y., Martins, E., Groboillot, A. and Neufeld, R. J. Spectrophotometric quantification of lactic bacteria in alginate and control of cell release with chitosan coating. J. Appl. Microbiol., 84, 342-348 (1998). 